\title{
Galaxy formation and evolution with the Dark Energy Survey
}

\author{
Diego Capozzi ${ }^{1}$, Daniel Thomas ${ }^{1}$, \\ Claudia Maraston ${ }^{1}$ and Luke J. M. Davies ${ }^{1,2}$ \\ ${ }^{1}$ Institute of Cosmology and Gravitation, University of Portsmouth \\ Dennis Sciama Building, Burnaby Road \\ Portsmouth, PO1 3FX, UK \\ email: diego.capozzi@port.ac.uk \\ email: daniel.thomas@port.ac.uk \\ email: claudia.maraston@port.ac.uk \\ ${ }^{2}$ Department of Physics, University of Bristol \\ H. H. Wills Physics Laboratory, Tyndall Avenue \\ BS8 1LT, UK \\ email: luke.davies@bristol.ac.uk
}

\begin{abstract}
The Dark Energy Survey (DES) will be the new state-of the-art in large-scale galaxy imaging surveys. With $5,000 \mathrm{deg}^{2}$, it will cover an area of the sky similar to SDSS-II, but will go over two magnitudes deeper, reaching $24^{\text {th }}$ magnitude in all four optical bands (griz). DES will further provide observations in the redder $Y$-band and will be complemented with VISTA observations in the near-infrared bands $J H K$. Hence DES will furnish an unprecedented combination of sky and wavelength coverage and depth, unreached by any of the existing galaxy surveys. The very nature of the DES data set - large volume at intermediate photometric depth - allows us to probe galaxy formation and evolution within a cosmic-time range of $\sim 10 \mathrm{Gyr}$ and in different environments. In fact there will be many galaxy clusters available for galaxy evolution studies, given that one of the main aims of DES is to use their abundance to constrain the equation of state of dark energy. The X-ray follow up of these clusters, coupled with the use of gravitational lensing, will provide very precise measures of their masses, enabling us to study in detail the influence of the environment on galaxy formation and evolution processes. DES will leverage the study of these processes by allowing us to perform a detailed investigation of the galaxy luminosity and stellar mass functions and of the relationship between dark and baryonic matter as described by the Halo Occupation Distribution.
\end{abstract}

Keywords. Astronomical data bases: surveys, galaxies: abundances, galaxies: formation, galaxies: evolution, galaxies: clusters: general, cosmology: observations

\section{Introduction}

In the past decades, the astrophysical community has put much effort into understanding how galaxies and the structures in which they assemble over cosmic time, form and cluster together. Within the currently accepted standard cosmological model, Dark Matter and Dark Energy (both of them only having been indirectly detected) play a fundamental role, which can be inferred by the outstanding results obtained by comparing the predictions of N-body simulations (e.g., Springel et al. 2005; Klypin et al. 2011) - which aim at reproducing the Universe's dark matter distribution - with observations performed with large galaxy surveys like the Sloan Digital Sky Survey (SDSS), using galaxies as dark matter tracers (e.g., Springel et al. 2006). The current picture predicts that structures (or haloes) form hierarchically due to the dark matter clustering. However, the picture portrayed for dark matter might not be applicable to baryonic 
matter (constituting the galaxy stellar and gas content), because it is not subject only to gravity. In fact, despite the general belief that galaxies form hierarchically - most of semi-analytic models are built on this premise (e.g., De Lucia et al. 2006) - several are the observations this scenario struggles to reproduce (e.g., downsizing, see Cowie et al. 1996; Thomas et al. 2005; Capozzi et al. 2010, Pozzetti et al. 2010 and references therein). Furthermore, the influence of the physics driving galaxy formation on galaxy-structure properties is still unresolved (e.g., Peacock \& Smith 2000; Berlind \& Weinberg 2002; Lin et al. 2004; Capozzi et al. 2012). The Dark Energy Survey (DES) will cover a niche of medium depth with a sky coverage that will allow us to probe the build-up of galaxy mass, and the dark-baryonic matter relation in galaxy structures out to $z \sim 1.3$.

\section{Abundance of massive galaxies}

The way the massive $\left(\gtrsim 10^{11} \mathrm{M}_{\odot}\right)$ end of the stellar mass function (SMF) builds up over cosmic time is currently a matter of debate. On the one hand, theoretical (semianalytic) models (e.g., De Lucia et al. 2006; De Lucia \& Blaizot 2007), constructed on the hierarchical structure build-up, predict that this part of the SMF evolved significantly in the last $\sim 10$ Gyr, during which galaxies accreted between 20 and $50 \%$ of their final mass; on the other hand, observations hint at a mass-dependent galaxy number-density evolution with $z$, i.e. the higher the mass the less the number-density evolution (Cimatti et al. 2006; Pozzetti et al. 2010). These discrepancies between observations and theoretical models put uncertainties on galaxy formation, which could be reduced by having a survey able to access a volume large enough to detect a representative sample of the most massive galaxies in the Universe (the rarest part of the galaxy population), and deep enough to identify a mass-complete galaxy sample out to $z \gtrsim 1$. The recent results of the Baryonic Oscillations Spectroscopic Survey (Maraston et al. 2012) at $0.4<z<0.7$ (a crucial range for the late mass build up predicted theoretically) showed a mild evolution of the massive end of the SMF. However, the $z$ range explored was too narrow to include the highest- $z$ window necessary for fully probing the mass build-up process. Studying the SMF with the DES dataset will allow us to do so, given its outstanding photometric quality, depth and spanned volume (the surveyed area will be 5,000 sq. deg).

\section{Detection of massive galaxies at $z>3$}

An intriguing byproduct of the DES will be the identification of very massive high redshift $(z>3)$ galaxies (Davies et al. 2012). Cold Dark Matter simulations (scaled to DES accessible volume) predict that no massive $\left(M>10^{12} \mathrm{M}_{\odot}\right)$ sources should exist at $z>3$, while passively evolving the low- $z$ mass function predicts such sources should be rare but nonetheless present at early times. However - given the large integration time and accessible volume needed to identify high- $z$ sources - rare, massive $(M>$ $10^{12} \mathrm{M}_{\odot}$ ) galaxies, which may be already passively evolving at $z \sim 3$, have probably missed detection in past and current surveys (Fig. 1). DES depth and sky coverage will allow the identification of such galaxies, should they exist, helping us to constrain galaxy formation models. By modeling (Davies et al. 2012) these sources we find that they must be sufficiently young, massive and relatively dust free, to be detected.

\section{Halo occupation distribution over cosmic time}

DES will detect a large number of galaxy clusters out to $z \sim 1.3$. Hence, it will provide a unique opportunity to study the evolution of the Halo Occupation Distribution 
Figure 1. Figure from Davies et al. (2012). Comparison of $z$-band magnitude limit vs. area for a sample of surveys. The grey region is the parameter-space area which is likely to detect $M>10^{12} \mathrm{M}_{\odot}$ galaxies at $z>3$. DES falls in this region as it covers a large area and is relatively deep in $z$.

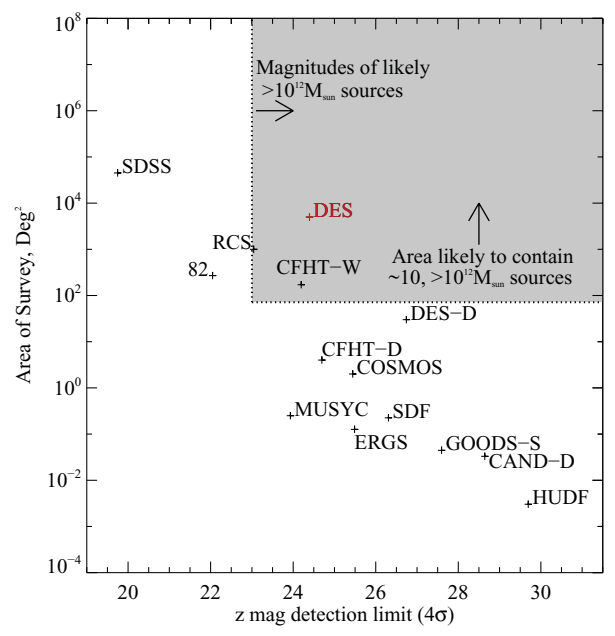

(HOD, Peacock \& Smith 2000; Berlind \& Weinberg 2002; Kravtsov et al. 2004) over cosmic time. Within the halo model, we focus on two aspects of the HOD framework: i) the richness-mass $(N-M)$ relation; ii) the relation between galaxy and dark matter spatial distributions within haloes, as parametrized by the density-profile concentration parameter $(c)$.

i) For haloes with masses $>10^{13} \mathrm{M}_{\odot}$ (galaxy groups and clusters), the $N-M$ relation can be approximated by a power law, $N \propto M^{\beta}$. The $\beta$ parameter at low $z$ has been deeply investigated in the literature (e.g., Lin et al. 2004; Muzzin et al. 2007) and the majority of the results point at a value $\beta<1$. This means that more massive structures have a lower number of galaxies per unit mass compared to their less massive counterparts, a fact that could be due to galaxy structures being less rich at high $z$. By studying a sample of some of the highest- $z(0.8<z<1.5)$ X-ray-selected clusters (Capozzi et al. 2012), we found evidence that clusters were indeed poorer in the past compared to local ones of the same mass (Fig. 2, left panel). However, this fact cannot be considered as the cause of the local $\beta<1$, unless corroborated by a significant evolution of the latter towards a value of 1 at high $z$. This possibility could be studied only by using mock clusters, with our results showing $\beta$ remaining $\sim 0.8$ between $0<z<1$. 3. However, a detailed study of this issue should be carried out observationally over a wide $z$ range.

ii) The importance of $c$ resides in its relation with structure formation time (Navarro et al. 1997; Neto et al. 2007; Gao et al. 2008 ). However, this somewhat direct correspondence is driven by gravity only. Differences between the dark-matter measured $c$ and the one measured using baryonic matter (e.g., galaxies or intra-cluster medium) could break this correspondence and shed light on the influence that galaxy formation has on the formation and evolution of structures over comic time. Hints of such differences (Fig. 2, right panel) have been found using simulations (Capozzi et al. 2012). However this result could not be associated with a physical process, nor could it be probed observationally, due to the lack of adequate data-sets.

Due to the heterogeneity of the analysis methods and cluster-sample characteristics, the observation-based studies in the literature also provide a blurred picture of the HOD evolution with $z$, leaving us with the need of a systematic and homogeneous study of $N-M$ and $c$ to be carried out on a statistically-rich cluster sample over a large $z$-range (out to $z \gtrsim 1$ ). DES will provide such a sample. 

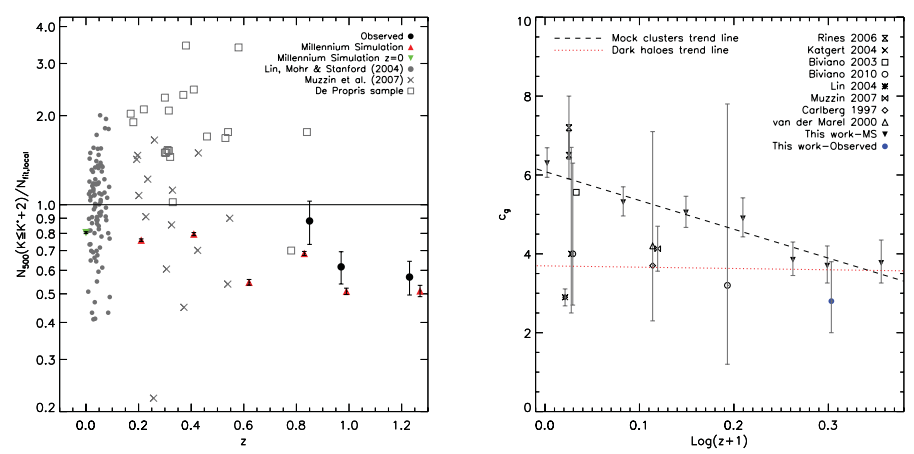

Figure 2. Figures from Capozzi et al. (2012) (see references therein). Left panel: ratio of measured $N_{500}$ over the prediciton of the low- $z$ best-fitting relation of Lin et al. (2004), vs. $z$. Right panel: average galaxy $c$ vs. $\log (z+1)$. Data points are observations, except full downward triangles (mock clusters in Capozzi et al. 2012). Dashed and dotted lines are trend lines for mock clusters and haloes with same mass by Capozzi et al. (2012) and Gao et al. (2008).

\section{Conclusions}

With its characteristics - area of $5,000 \mathrm{deg}^{2}$; depth of $\sim 24^{\text {th }}$ in four optical bands (griz) - DES will allow us to study in detail the galaxy mass build-up process since $z \sim 1.3$ and to possibly detect, should they exist, $M>10^{12} \mathrm{M}_{\odot}$ galaxies at $z>3$. This will help us to constrain galaxy formation models. Finally, the large number of galaxy clusters that DES will detect between $0<z<1.3$ will allow us to study the prospective evolution of the HOD in detail, hence giving us the chance to probe the relation between dark and baryonic matter over such a large redshift range.

\section{References}

Berlind, A. A. \& Weinberg, D. H. 2002, ApJ, 575, 587

Capozzi, D., Collins, C. A., \& Stott, J. P. 2010, MNRAS, 403, 1274

Capozzi, D., Collins, C. A., Stott, J. P., \& Hilton, M. 2012, MNRAS, 419, 2821

Cimatti, A., Daddi, E., \& Renzini, A. 2006, A\& $\mathcal{E}$, 453, L29

Cowie, L. L., Songaila, A., Hu, E. M., \& Cohen, J. G. 1996, AJ, 112, 839

Davies, L. J. M., Maraston, C., Thomas, D., \& Capozzi, D., the DES collaboration 2012, submitted

De Lucia, G., Springel, V., White, S. D. M., Croton, D., \& Kauffmann, G. 2006, MNRAS, 366, 499

De Lucia, G. \& Blaizot, J. 2007, MNRAS, 375, 2

Gao, L., et al. 2008, MNRAS, 387, 536

Klypin, A. A., Trujillo-Gomez. S., \& Primack, J. 2011, ApJ, 740, 102

Kravtsov, A. V., et al. 2004, ApJ, 609, 35

Lin, Y., Mohr, J. J., \& Stanford, S. A. 2004, ApJ, 610, 745

Maraston, C., et al. 2012, MNRAS, submitted (arXiv:1207.6114)

Muzzin, A., Yee, H. K. C., Hall, P. B., \& Lin, H. 2007, ApJ, 663, 150

Navarro, J. F., Frenk, C. S., \& White, S. D. M. 1997, ApJ, 490, 493

Neto, A. F., et al. 2007, MNRAS, 381, 1450

Peacock, J. A. \& Smith, R. E. 2000, MNRAS, 318, 1144

Pozzetti, L., et al. 2010, A\&SA, 523, A13

Springel V., et al. 2005, Nature, 435, 629

Springel, V., Frenk, C. S., \& White, S. D. M. 2006, Nature, 440, 1137

Thomas, D., Maraston, C., Bender, R., \& Mendes de Oliveira, C. 2005, ApJ, 621, 673 